\title{
Análisis de la Cumplimentación de Certificados Médicos por Estudiantes y Profesionales de Odontología
}

\author{
ANALYSIS OF FILLING OUT DENTAL CERTIFICATES BY DENTAL STUDENTS AND PROFESSIONALS
}

Cléa Adas Saliba Garbin'1, Ronald Jefferson Martins', Naiana de Melo Belila', Tânia Adas Saliba', Artênio José İ́sper Garbin ${ }^{1}$

1. Facultad de Odontología de Araçatuba, UNESP - Univ Estadual Paulista, Araçatuba, São Paulo, Brasil.

\begin{abstract}
RESUMEN
Este estudio tuvo el objetivo de analizar y comparar la cumplimentación de certificados médicos por estudiantes del cuarto año del curso de Odontología de una universidad pública brasileña y por profesionales de cursos de especialización específicos del área. Entre 2014 y 2016, se dejó a los encuestados una hoja de papel en blanco en cuyo encabezamiento sólo constaba la palabra "Certificado", a fin de que se lo elaboraran de la manera que juzgaran más adecuada. Se utilizó test para comparación de dos proporciones y Test de ChiCuadrado con nivel de significación del 5\%. Participaron de la investigación 336 individuos, de los cuales 167 (49,7\%) eran estudiantes y 169 (50,3\%), profesionales de cursos de especialización de la Facultad de Odontología de Araçatuba. Solamente 73 (21,7\%) individuos elaboraron el certificado correctamente. Entre los errores de los estudiantes se destaca la inserción de la Clasificación Estadística Internacional de Enfermedades sin el consentimiento del paciente, y, de los profesionales, la falta del Documento Nacional de Identidad. Hubo diferencia estadística significativa entre las categorías respecto a la correcta cumplimentación del certificado $(\mathrm{p}=0)$. Se concluyó que una parte considerable de los encuestados rellena los certificados odontológicos incorrectamente, con menores errores entre los estudiantes de Odontología.
\end{abstract}

(Saliba Garbin C, Jefferson R, Belila N, Saliba T, Ísper A, 2018. Análisis de la Cumplimentación de Certificados Médicos por Estudiantes y Profesionales de Odontología. Cienc Trab. Ene-Abr; 20 [61]: 27-30).

Palabras clave: CERTIFICADO DE SALUD, DOCUMENTACIÓN, PRÁCTICA PROFESIONAL.

\section{ABSTRACT}

The objective of this study was to analyze and compare the filling of the dental certificates by the students of the fourth year of the dentistry course of a Brazilian public university and professionals of specialization courses in the area. The respondents were given a blank sheet written "Certificate", for them to fill out. The period analyzed was from 2014 to 2016. The test was used to compare two proportions and the chi-square test, with a significance level of 5\%. A total of 336 individuals participated in the study, of which 167 (49.7\%) were fourth year students of the Dentistry course and 169 (50.3\%) professionals of specialization courses of the School of Dentistry of Araçatuba. Only $73(21.7 \%)$ correctly filled the certificate. Among the errors, we emphasize the students' insertion of the International Statistical Classification of Diseases without the patient's consent and among professionals the lack of the General Registry. There was a significant statistical difference between the categories regarding the correct filling of the certificate $(p=0)$. We concluded that the dental certificates are filled incorrectly by a considerable portion of the respondents, with minor errors among dentistry students.

Key words: HEALTH CERTIFICATE, DOCUMENTATION, PROFESSIONAL PRACTICE.

\section{INTRODUCCIÓN}

Los historiales clínicos odontológicos comprenden un conjunto de declaraciones firmadas por profesional y paciente y sirven como prueba en circunstancias jurídicas. Abarcan anamnesis, consentimiento libre y aclarado, evolución clínica del tratamiento, fotogra-

Correspondencia / Correpondence:

Cléa Adas Saliba Garbin

NEPESCO - Núcleo de Pesquisa em Saúde Coletiva

Facultad de Odontología de Araçatuba, UNESP - Univ Estadual Paulista

Departamento de Odontología Infantil y Social

Calle José Bonifácio, 1193 - Barrio Vila Mendonça

CP 16015-050 - Araçatuba - São Paulo - Brasil

Tel.: (55 18) 3636-2824

e-mail: cgarbin@foa.unesp.br

Recibido: 18 de noviembre de 2017 / Aceptado: 13 de Enero de 2018

fías y radiografías, así como copias de recetas, certificados y otros documentos del paciente. ${ }^{1}$

El certificado es una declaración particular, sucinta y por escrito, por la que el cirujano-dentista declara que el paciente estuvo bajo sus cuidados profesionales y sus consecuencias, conllevando providencias administrativas y judiciarias relacionadas al paciente. ${ }^{2}$ Los certificados médicos y odontológicos son los únicos instrumentos legales que sirven para justificar las faltas al trabajo por motivo de enfermedad y asegurar el pago del sueldo. ${ }^{3}$

La autenticidad de los hechos certificados es crucial, pues el cirujano-dentista puede sufrir sanciones si infringe el artículo 299, falsedad ideológica, del Código Penal Brasileño. ${ }^{4}$ Puede también estar sujeto a pena y procesos éticos impuestos por el Consejo Regional de Odontología, una vez que el Código de Ética Odontológico establece en su artículo 7 como infracción ética: XI"proporcionar certificado que no corresponda a la veracidad de los hechos o de los cuales no haya participado." 5,6

De acuerdo con el artículo 33 de ese código, los certificados odon- 
tológicos y cualquier otro tipo de impreso odontológico deben contener el nombre completo del profesional, la profesión, en ese caso cirujano-dentista, el número de registro en el CR05, y se deben expedir en dos copias, perteneciendo la segunda al archivo del historial clínico del paciente. ${ }^{1,7}$

Ocasionalmente, los acompañantes también necesitan certificado. En esos casos, se debe emitir una declaración de comparecencia al acompañante del menor de edad. ${ }^{1}$

Dentro de ese contexto, este estudio tuvo como objetivo analizar y comparar la cumplimentación de certificados odontológicos por estudiantes del cuarto año del curso de Odontología de una institución de enseñanza superior y por profesionales de cursos de especialización de esa área.

\section{METODOLOGÍA}

Esta investigación es un estudio transversal, descriptivo y cuantitativo. En el período de 2014 a 2016 se analizó la cumplimentación de certificados odontológicos por estudiantes del cuarto año del curso de Odontología y profesionales de cursos de especialización de una institución pública de enseñanza superior.

Se dejó a los encuestados una hoja de papel en blanco en cuyo encabezamiento solo constaba la palabra "Certificado", a fin de que se elaboraran un certificado odontológico de la manera que juzgaran más adecuada.

Para el análisis de las informaciones recolectadas, se utilizó una hoja de cálculo del programa Microsoft Excel especialmente desarrollada para este estudio. Los resultados se presentaron en frecuencias absolutas y porcentuales.

Para el análisis estadístico se realizaron el test para comparación de dos proporciones y el Test de Chi-Cuadrado, con nivel de significación del 5\%, utilizando el programa BioStat 5.0.8

La investigación fue aprobada por el Comité de Ética en Investigación en Seres Humanos de la Facultad de Odontología de Araçatuba/UNESP, obedeciendo las normas éticas dispuestas en la Resolución 466/2012 del Consejo Nacional de Salud del Ministerio de Salud del Brasil. Todos los participantes firmaron el Término de Consentimiento Libre y Aclarado.

\section{RESULTADOS}

Participaron de la investigación 336 individuos, de los que 167 $(49,7 \%)$ eran estudiantes del cuarto año del curso de Odontología y 169 (50,3\%), profesionales de cursos de especialización de la Facultad de Odontología de Araçatuba.

Del total de encuestados, solo 73 (21,7\%) realizaron la cumplimentación correcta del certificado. Se notó que la gran mayoría de ambas categorías cumplimentó de manera incorrecta el certificado, siendo que los estudiantes tuvieron mayor número de aciertos, con diferencia estadística significativa, conforme el gráfico 1. Además, solamente $4(1,2 \%)$ cumplimentaron dos copias del certificado.

Una parte significativa, $126(37,5)$ de los participantes informó la Clasificación Estadística Internacional de Enfermedades y Problemas Relacionados con la Salud (CIE-10) en el certificado odontológico, aunque no estaba escrito el término "a pedido del interesado".9 Mismo sin permisión de identificación del procedimiento ejecutado durante el tratamiento odontológico, 26 (7,7\%) participantes lo describieron en el certificado.

\section{Gráfico 1.}

Análisis comparativo de los certificados odontológicos de acuerdo con la forma de cumplimentación por estudiantes y profesionales de Odontología, Araçatuba, 2017.

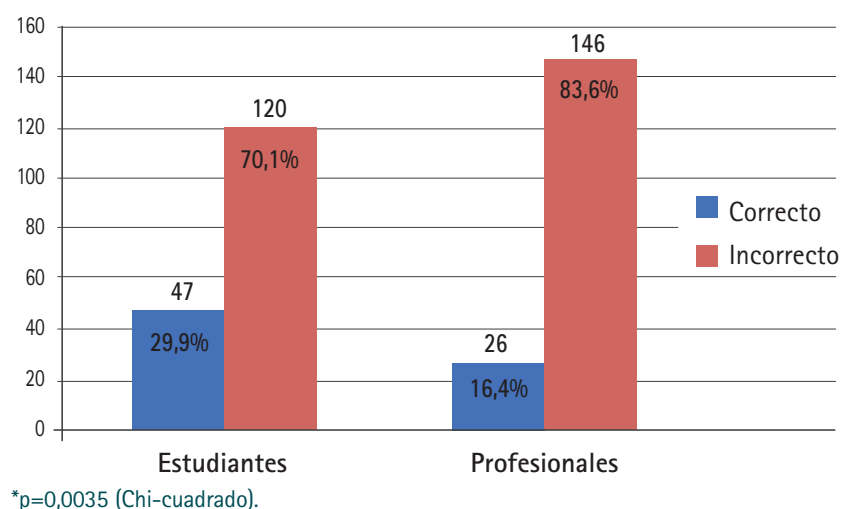

Gráfico 2.

Análisis comparativo de los certificados odontológicos de acuerdo con la cumplimentación incorrecta de los datos por estudiantes y profesionales de Odontología, Araçatuba, 2017.

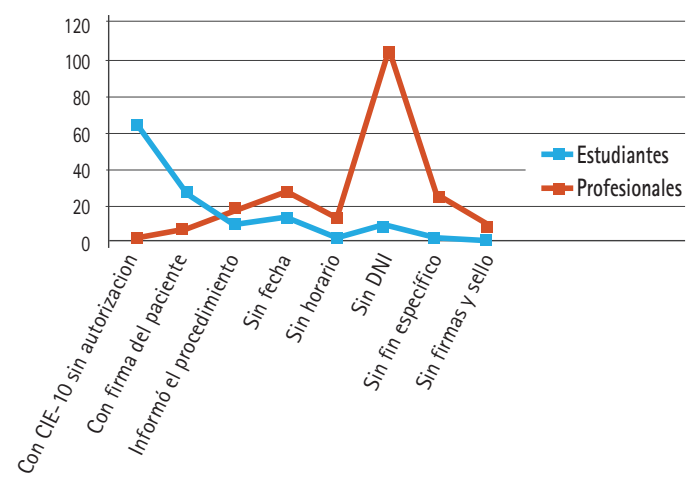

Durante la cumplimentación, más de la mitad de los encuestados $(60,1 \%)$ no informó uno o más tipos de datos importantes como: fecha de la descripción del certificado (11,9\%); el horario de inicio y término de la atención (4,2\%); el DNI del paciente o del responsable/ acompañante (33,9\%); el número de registro en el CRO o sello, incluso la firma $(2,4 \%)$; y el fin específico del certificado (laboral, escuela y otros) (7,7\%). También, 194 (57,7\%) informaron el término "para los fines pertinentes" y $33(9,8 \%)$ solicitaron la firma del paciente en la primera copia del certificado.

Cuando se observa la cumplimentación por los estudiantes, se nota mayor incorrección al informar el CIE-10 sin obtener la autorización del paciente. Ya en los profesionales de Odontología, se observa la falta del DNI del paciente (Gráfico 2).

En la tabla 1, se observa la asociación entre la forma de cumplimentación de los datos y la categoría encuestada.

\section{DISCUSIÓN}

Los certificados odontológicos son documentos legales cuya redacción y forma de ofrecimiento deben ser cuidadas por el cirujanodentista a fin de que se eviten problemas judiciales. ${ }^{1}$

Legalmente, la Ley 5.081, de 24 de agosto de $1966^{10}$, la que regula el ejercicio profesional, dispone en su artículo sexto, párrafo III, como 
Tabla 1.

Número, porcentaje y $\mathrm{p}$ valor de acuerdo con la cumplimentación de los datos por estudiantes y profesionales de Odontología, Araçatuba, 2017.

\begin{tabular}{lcccccccccc} 
& \multicolumn{4}{c}{ Estudiantes } & \multicolumn{6}{c}{ Profesionales } \\
İtems & \multicolumn{2}{c}{ Cierto } & Errado & \multicolumn{2}{c}{ Cierto } & Errado & \\
cumplimentados & $\mathrm{n}$ & $\%$ & $\mathrm{n}$ & $\%$ & $\mathrm{n}$ & $\%$ & $\mathrm{n}$ & $\%$ & $\mathrm{P}$ valor \\
ClE-10 con autorización & 102 & 61,1 & 65 & 38,9 & 169 & 100 & 0 & 0 & $<0,0001$ \\
Firma del paciente & 141 & 84,4 & 26 & 15,6 & 162 & 95,9 & 7 & 4,1 & $=0,0004$ \\
Informó el procedimiento & 158 & 94,6 & 9 & 5,4 & 152 & 89,9 & 17 & 10,1 & $=0,1092$ \\
Fecha & 154 & 92,2 & 13 & 7,8 & 142 & 84,0 & 27 & 16 & $=0,0204$ \\
Horario & 166 & 99,4 & 1 & 0,6 & 156 & 92,3 & 13 & 7,7 & $=0,0011$ \\
DNI del profesional & 159 & 95,2 & 8 & 4,8 & 63 & 37,3 & 106 & 62,7 & $<0,0001$ \\
Fin específico & 166 & 99,4 & 1 & 0,6 & 144 & 85,2 & 25 & 14,8 & $<0,0001$ \\
Firma y sello & 167 & 100 & 0 & 0 & 161 & 95,3 & 8 & 4,7 & $=0,0044$ \\
\hline Cumplimentación de & & & & & & & & & \\
los certificados & 47 & 28,1 & 120 & 71,9 & 26 & 15,4 & 146 & 84,6 & $=0,0035$
\end{tabular}

siendo de competencia del cirujano-dentista "atestiguar, en el sector de su actividad profesional, estados mórbidos y otros”. Sin embargo, fue solamente el 30 de junio de 1975 que la Ley 6.215 cambió la redacción de ese ítem a: artículo sexto, párrafo III - "atestiguar, en el sector de su actividad profesional, estados mórbidos y otros, incluso para justificar ausencias laborales". ${ }^{11}$ Esa alteración reconoce la importancia del estado mórbido de competencia odontológica como factor de absentismo. ${ }^{12}$

El profesional deberá emitir el certificado exclusivamente en caso concreto de atención, una vez que la ley no permite la emisión del documento por motivo gratuito, o sea, sin que el paciente no tenga el problema de salud. La falta de veracidad en sus afirmaciones podrá ocasionar al profesional imputación de falsedad ideológica, crimen previsto en el artículo 299 del Código Penal Brasileño (Ley 2.848): Pena - reclusión de uno a tres años. Si se comete el crimen con fines de lucro, se le aplica al profesional también una multa. ${ }^{4}$ Para la correcta cumplimentación de un certificado odontológico, se debe dividirlo en algunas partes. La primera es aquella relativa a las cualificaciones del profesional, la que ya es parte del recetario impreso y en el que se va a redactar el certificado. ${ }^{13}$

En la segunda parte deberá constar la calificación del paciente, su identificación y la finalidad a que se destina el certificado, es decir, para fines laborales, escolares, deportivos o militares, pudiendo incluirse la información de que el certificado se elaboró a pedido del interesado. Se destaca que, segundo el CEO - Centro de Especialidades Odontológicas -, no se debe usar el término genérico "para los fines pertinentes".,14 En este estudio, una parte de los encuestados no informó la finalidad del certificado y, así, utilizó dicho término.

Tras haber informado todos esos datos, el cirujano-dentista aun deberá declarar en el certificado que el paciente estuvo bajo sus cuidados profesionales, sin especificar la naturaleza de la atención. Si hay la necesidad de que se exponga el diagnóstico, como por ejemplo, por exigencia del empleador, deberá utilizarse la CIE-10 delante autorización y firma del paciente. Aún así no es permitido revelar los procedimientos ejecutados.

Hasta la quinta revisión de la CIE, sólo se le añadían nuevas enfermedades si ésas causaban muerte. Después de la sexta revisión, la CIE se tornó una clasificación que incluye todas las enfermedades y no solamente aquellas que llevan a la muerte. ${ }^{15}$

La resolución 1.819/2007 del Consejo Federal de Medicina prohíbe la inclusión de la CIE-10 en los certificados médicos en casos de que puedan provocar prejuicio. El artículo 102 del Código de Ética
Médica afirma que el médico no puede revelar la enfermedad del paciente sin su autorización previa. Así se entiende que el certificado se deberá aceptar en las empresas con o sin la CIE-10. ${ }^{16} \mathrm{El}$ departamento de recursos humanos de las empresas deberá estar fundamentado por esa legislación, a fin de que la empresa no tenga problemas judiciales de daños morales por no aceptar los certificados médicos u odontológicos de sus empleados.

Una parte de los encuestados describió el tipo de procedimiento ejecutado durante el tratamiento odontológico del paciente e informó la CIE-10 sin hacer constar el término "a pedido del interesado". En la investigación de Garbin y colaboradores ${ }^{12}$, se observó que la mayoría de los cirujanos-dentistas describía en el certificado el acto practicado. Ya en otro estudio, gran parte no informó la CIE-10 referente al motivo de la ausencia al trabajo, sugiriendo que ese hecho puede haber causado la no aceptación del certificado por la empresa. ${ }^{3}$

Esas evidencias sugieren el desconocimiento sobre el secreto profesional, o de la CIE-10, el que se estableció para que los profesionales del área de la salud pudieran comunicarse sin describir el tratamiento realizado y sin romper el sigilo profesional. ${ }^{17}$

A continuación, el certificado debe tener una breve conclusión referente a sus consecuencias (imposibilidad de comparecencia al trabajo; que el paciente estuvo bajo sus cuidados profesional dentro de un límite de hora; y sobre la necesidad de reposo por determinado tiempo). La fecha y el horario de atención al paciente deben ser exactos. En esta investigación, parte de los encuestados no informó el horario de tratamiento del paciente, lo que puede afectar el profesional de Odontología en caso de que ocurra algún acto ilícito por parte del paciente en el día certificado. El profesional puede ser considerado cómplice del acto y responder en juicio por él.

Finalmente, todo documento debe tener local y fecha de emisión, firma del cirujano-dentista responsable, nombre del profesional, profesión y número del registro en el CRO impresos por un sello. ${ }^{17}$ Es necesario hacer copia carbonada o emisión en dos copias con rúbrica del paciente en la segunda, la que será archivada en su historial. ${ }^{17}$ En este estudio, una ínfima parte de los encuestados emitió la segunda copia del certificado, y muchos solicitaron que el paciente firmara la primera copia, justo la que será del proprio paciente.

Se observaron diversos errores durante la cumplimentación de los certificados por estudiantes y profesionales. Sin embargo, los profesionales de Odontología tuvieron mayor número de cumplimentaciones incorrectas, lo que sugiere un hábito desfavorable desarrollado a lo largo del tiempo de la práctica profesional, sea por prisa o desprecio a detalles del certificado. Los estudiantes, a su vez, ya habían tomado una asignatura en la que se les enseñaba la correcta redacción de las documentaciones odontológicas.

\section{CONCLUSION}

Se concluye que una parte considerable de los encuestados cumplimentaron los certificados odontológicos de manera incorrecta. Comparativamente, los estudiantes de Odontología se equivocaran menos en la cumplimentación que los profesionales.

La negligencia de los cirujanos-dentistas en la cumplimentación de esos documentos es preocupante, pues puede, asimismo, llevarlos a juicio. 


\section{REFERENCIAS}

1. Amorim HPL, Marmol SLP, Cerqueria SNN, Silva MLCA, Silva UA. A importância do preenchimento adequado dos prontuários para evitar processos em Odontologia. Arq Odontol, 2016; 52(1):32-37.

2. Garbin CAS, Garbin AJl, Lelis RT. Verificação das atitudes de cirurgiõesdentistas quanto à documentação de seus pacientes. Rev Assoc Paul Cir Dent, 2006; 60(6):442-445.

3. Martins RJ, Garbin CAS, Garbin AJI, Moimaz SAS. Absenteísmo por motivos odontológico e médico nos serviços público e privado. Rev Bras Saúde Ocup. 2005; 30(111):9-15

4. Brasil. Código penal, Decreto-Lei $n^{\circ}$ 2.848. Diário Oficial da União, Seção 1 (31 dez 1940): 23911 (Publicação Original) [on line]. [acesso 20 jul 2017]. Disponivel em: http://www2.camara.leg.br/legin/fed/declei/1940-1949/ decreto-lei-2848-7-dezembro-1940-412868-publicacaooriginal-1-pe.html

5. Brasil. Conselho Federal de Odontologia. Código de Ética Odontológica; aprovado pela Resolução CFO-118 (2012) [on line]. São Paulo: CRO; 2012 [acesso 20 jul 2017]. Disponivel em: http://www.crosp.org.br/crosp_v4/ uploads/etica/2b1ef1e3329d8f7902ce0f52e536a89c.pdf.

6. BRASIL. Conselho Federal de Odontologia. Prontuário odontológico: uma orientação para o cumprimento da exigência contida no inciso VIII do art. $5^{\circ}$ do Código de Ética Odontológica [on line]. Rio de Janeiro: CFO; 2004 [citado 20 jul 2017]. Disponivel em: http://www. cfo.org.br.

7. França BS. Atestados odontológicos: cuidados na redação. Rev Clín Ortodon Dental Press. 2007; 6(3):44-45.

8. Ayres $M$, Ayres Jr M, Ayres DL, Santos AS. BioEstat 5.0: aplicações estatísticas nas áreas das ciências biomédicas [programa de computador]. Belém: ONG Mamieraua; 2007.
9. Organização Mundial de Saúde. CID-10: Classificação estatística internacional de doenças e problemas relacionados à saúde. 5a ed. São Paulo: Edusp; 1997. 1191 p.

10. Brasil. Regula o exercício da Odontologia, Lei $n^{\circ}$ 5.081. Diário Oficial da União, Brasilia (24 agos 1966).

11. Brasil. Legislação Informatizada-Dados da Norma, Lei $n^{\circ}$ 6.215. Diário Oficial da União, Brasília, Seção 1 (1 jul 1975): 7897 (Publicação Original) [on line]. [citado 15 jul 2017]. Disponivel em: http://www2.camara.leg.br/legin/fed/ lei/1970-1979/lei-6215-30-junho-1975-357631-norma-pl.html

12. Garbin CA et al. 0 cirurgião-dentista e a emissão de atestados odontológicos. Odontol Soc. 2000; 2(1-2):89-92.

13. Peres AS, Peres SHCS, Silva RHA, Ramires I. 0 novo código de ética odontológica e atuação clínica do cirurgião-dentista: uma reflexão crítica das alterações promovidas. Rev Odontol Araçatuba. 2004; 25(2):9-13.

14. Silva M. Documentação em Odontologia e sua Importância Jurídica. Odontol Soc. 1999; 1(1-2):1-3.

15. Laurenti R. Pesquisas na área de Classificação de Doenças. Saúde Soc. 1994; 3(2):112-126.

16. Brasil. Conselho Federal de Medicina. Resolução CFM n 1.819 (17 maio 2007) [on line]. Brasilia: Portal médico.com; 2007 [citado 12 ago 2017]. Disponivel em: http://www.portalmedico.org.br/resolucoes/cfm/2007/1819 2007.htm

17. Paranhos LR, Salazar M, Ramos AL, Siqueira DF. Orientações legais aos cirurgiões dentistas. Rev Odonto (São Paulo). 2007; 15(30):55-62.

- Carrion V. Nova jurisprudência em direito do trabalho. São Paulo: Revista dos Tribunais; 1994. $702 \mathrm{p}$. 